\title{
PERCEPÇÃO DAS GESTANTES AO PRÉ-NATAL E COMPLICAÇÕES DURANTE O PERÍODO GRAVÍDICO-PUERPERAL
}

\section{Perception of pregnant women in prenatal and complications during the pregnancy-puerperal period}

\footnotetext{
https://doi.org/10.18593/eba.28144

Recebido em 24 de julho de 2021 | Aceito em 17 de novembro de 2021

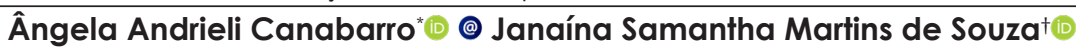

Graduanda em Enfermagem pela Faculdade Nossa Senhora de Fátima; Rua Alexandre Fleming, $\mathrm{n}^{\circ} 454$ - Bairro Madureira, Caxias do Sul RS, 95041-520; https://orcid.org/0000-0001-9919-4746; angelacanabarro@gmail.com

Doutora em Medicina e Ciências da Saúde. Mestre em Saúde Coletiva.
}

Resumo: Introdução - O pré-natal se realizado corretamente desde o início da gestação é fundamental para identificar precocemente possíveis patologias, anomalias congênitas e complicações no decorrer do período gestacional. Objetivo - Identificar a percepção das gestantes quanto ao pré-natal, correlacionando com as complicações durante a gestação. Métodos - Foi realizada a pesquisa com 30 gestantes do último trimestre gestacional de uma unidade básica de saúde, localizada em uma cidade do interior do Rio Grande do Sul. Em seguida os dados foram analisados por meio da estatística descritiva, com frequência relativa e absoluta. Para isso, utilizou-se o software SPSS versão 21. Resultados - Os achados presentes neste estudo destacam a adesão das gestantes ao pré-natal, desde o início da gestação. Conclusões - A qualidade da assistência prestada pela equipe de saúde, foi crucial para a percepção e a adesão das gestantes ao programa de pré-natal na atenção básica, contribuindo de forma positiva para a detecção precoce de possíveis complicações durante o período gravídico-puerperal.

Palavras-chave: Gestação. Pré-natal. Complicações. Enfermagem.

Abstract: Introduction - If performed correctly since the beginning of pregnancy, prenatal care is essential for the early identification of possible pathologies, congenital anomalies, and complications during pregnancy. Purpose Identify the perception of pregnant women about prenatal care, correlating with complications during pregnancy. Methods - The research was carried out with 30 pregnant women in the last trimester of pregnancy in a basic health unit, located in a city in the interior of Rio Grande do Sul. Then the data were analyzed using descriptive statistics, with relative and absolute frequency. For this, the SPSS software version 21 was used. Results - The findings present in this study highlight the adherence of pregnant women to prenatal care, from the beginning of gestation. Conclusions - The quality of care provided by the health team was crucial for the adherence of pregnant women to the prenatal program in primary care, contributing positively to the early detection of possible complications during the pregnancy-peruria period.

Keywords: Pregnancy. Prenatal. Complications. Nursing.

@ Autor correspondente: Graduanda em Enfermagem pela Faculdade Nossa Senhora de Fátima; Rua Alexandre Fleming, ${ }^{\circ} 454$ - Bairro Madureira, Caxias do Sul-RS, 95041-520; https://orcid.org/0000-0001-9919-4746; angelacanabarro@gmail.com 


\section{INTRODUÇÃO}

A assistência pré-natal tem por objetivo principal identificar de forma precocemente e adequadamente as patologias e riscos gestacionais, na qual acometem aquelas pacientes que têm mais chances de desenvolver algumas condições que tornam vulneráveis a saúde da mãe e do feto durante o período gestacional. Do mesmo modo acolhendo a gestante desde o início da sua gestação e lhe dando a devida atenção neste período gravídico puerperal' ${ }^{1}$.

Segundo Sehnem e colegas $^{2}$, a gestação é uma fase de grandes transformações na vida de uma mulher e de sua família, na medida que surgem várias alterações tanto fisiológicas como psicológicas, físicas e sociais. Neste momento as responsabilidades e novos papéis aumentam, fazendo com que surjam dúvidas, anseios, medos, frustrações, expectativas, insegurança, tanto para a gestante como para os demais familiares, principalmente se a gestante é primigesta.

Sendoassim nosúltimosanos foram criados vários programas com enfoque na assistência da saúde da mulher no Brasil, com ações e serviços que visam à promoção, prevenção da saúde e tratamento precoce de doenças, beneficiando essa população mais vulnerável, e buscando também diminuir a mortalidade materna e neonatal no país3.

A porta de entrada da gestante se dá exclusivamente pela atenção básica de saúde, sendo pertinente a atenção dedicada à área da saúde da mulher e da assistência ao prénatal. Tornando-se os cuidados da equipe multidisciplinar com objetivos principais de desfechos perinatais e maternos favoráveis, pois permite-se desenvolver precocemente os cuidados, condutas, e procedimentos em função da saúde da mãe e do feto, evitando assim possíveis complicações mais graves durante o período gravídico-puerperal e garantindo a saúde materna e fetal com qualidade, para assim reduzir os índices de morbimortalidade materna e fetal ${ }^{4}$.

Para Gomes e colegas 5 , o enfermeiro é um dos principais profissionais aptos para efetuar a assistência pré-natal, por estar qualificado para intervir em estratégias de promoção da saúde, prevenção de doenças, e por utilizara humanização nos cuidados prestados. Para tal, elabora o plano assistencial da consulta de acompanhamento do pré-natal, conforme as necessidades identificadas e priorizadas, estabelecendo assim às intervenções necessárias, orientações e encaminhando se necessário para os outros serviços de saúde, além de promover a interdisciplinaridade das ações.

É notável que a atenção ao pré-natal de qualidade é fundamental para reduzir a morbimortalidade materno infantil, sendo assim o atendimento à gestante no início do pré-natal é imprescindível, uma vez que facilita para um diagnóstico prévio de alterações e para a execução de ações adequadas em relação às condições que a grávida pode ser afetada ${ }^{6,7}$. Finalizando assim a assistência pré-natal com o nascimento de uma criança saudável e assegurando o bem-estar materno e neonatal.

À vista disso, levando em consideração a importância do tema abordado, este estudo teve por objetivo identificar a percepção das gestantes quanto ao pré-natal, correlacionando com as complicações durante a gestação, em uma unidade básica de saúde do município de Flores da Cunha, no Estado do Rio Grande do Sul. 


\section{MÉTODOS}

O presente estudo trata-se de uma pesquisa de campo exploratória, com abordagem quantitativa. Segundo o autor $\mathrm{Gil}^{8}$ a pesquisa exploratória tem como propósito fundamental resolver, expandir e alterar princípios ou convicções, seguindoa caracterizaçãodeproblemas mais concisos ou hipóteses pesquisáveis para aprendizados subsequentes. A coleta de dados foi realizada em uma unidade básica de saúde de atenção primária, localizada no município de Flores da Cunha/RS, no período de março a maio de 2021, durante o turno matutino, totalizando seis horas semanais.

Neste estudo foram incluídas 30 gestantes da $28^{\mathrm{a}}$ a $40^{\mathrm{a}}$ semana de gestação que realizavam o pré-natal pelo SUS nesta unidade básica de saúde, as quais concordaram em participar voluntariamente do estudo e assinaram o Termo de Consentimento Livre e Esclarecido (TCLE). Foram excluídos do estudo as gestantes menores de 18 anos e as que não concordaram em participar da pesquisa.

O questionário, elaborado com 30 perguntas abertas e fechadas, foi aplicado pela entrevistadora, no qual forneceu às gestantes após as consultas de acompanhamento do pré-natal. No momento da coleta de dados, as participantes foram orientadas quanto aos objetivos da pesquisa, sobre a importância do Termo de Consentimento Livre e Esclarecido. Deste modo, sem perdas ou desistências durante a pesquisa, após responderem as perguntas do questionário, foi garantido a confidencialidade de suas respostas. Em seguida, os dados foram lançados no programa Software Excel e analisados por meio da estatística descritiva, com frequência relativa e absoluta. Para isso, utilizou-se o software SPSS versão 21.

\subsection{APROVAÇÃO ÉTICA}

Este estudo tem a aprovação pelo Comitê de Ética em Pesquisa da Associação Cultural e Científica Virvi Ramos, sob o Parecer no ooo735/2021 (CAAE: 41732820.3.0000.5523). Os pesquisadores seguiram as diretrizes e normas regulamentadoras preconizadas na Resolução № 466/12 do Conselho Nacional de Saúde sobre as pesquisas envolvendo seres humanos.

\section{RESULTADOS}

A população estudada foi composta por 30 gestantes. A maioria delas possuem a idade entre 23 e 32 anos (66,7\%), são católicas (53,3\%), são brancas $(50 \%)$, tem ensino médio completo (50\%), possuem união estável (50\%) e renda de 1 a 3 salários mínimos $(66,7 \%)$. Em relação à profissão, há predominância por gestantes que são donas de casa $(23,3 \%)$, desempregadas $(20 \%)$ e auxiliar de produção (13,3\%). Os resultados estão na Tabela 1.

Tabela 1 - Caracterização da amostra. Unidade Básica de Saúde, Flores da Cunha, RS, Brasil, $2021(\mathrm{~N}=30)$.

\begin{tabular}{lcc}
\hline Idade & $\mathbf{n}$ & $\%$ \\
\hline 18 a 22 anos & 8 & 26,7 \\
23 a 27 anos & 9 & 30,0 \\
28 a 32 anos & 11 & 36,7 \\
33 anos ou mais & 2 & 6,7 \\
\hline Religião & & \\
\hline Católica & 16 & 53,3 \\
Evangélica & 11 & 36,7 \\
Testemunha de Jeová & 1 & 3,3 \\
Espírita & 2 & 6,7 \\
\hline
\end{tabular}




\begin{tabular}{lrc}
\hline Cor/raça & n & $\%$ \\
\hline Branca & & \\
Parda & 15 & 50,0 \\
Negra & 13 & 43,3 \\
\hline
\end{tabular}

\section{Escolaridade}

Ensino Fundamental Completo

Ensino Médio Incompleto

Ensino Médio Completo

Ensino Superior Incompleto

\section{Estado Civil}

\begin{tabular}{lcc}
\hline Casada & 9 & 30,0 \\
Solteira & 6 & 20,0 \\
União estável & 15 & 50,0 \\
\hline
\end{tabular}

\section{Renda}

Nenhuma renda $5 \quad 16,7$

Até 1 salário mínimo (até R\$ 1.045,0o) $2 \quad 6,7$

De 1 a 3 salários mínimos (de R $\$$ 1.045,00 a R\$3.135,00)

\section{Profissão}

Dona de casa $7 \quad 23,3$

Desempregada

Auxiliar de produção

Estudante

Vendedora

Operadora de caixa

Auxiliar de limpeza

Agricultora

Operadora financeira

Garçonete

Manicure

Cabelereira

Quanto ao comportamento durante a gestação, a pesquisa questionou se alguém na casa da gestante faz uso de álcool ou tabaco. Do total das 30 gestantes, $20(66,7 \%)$ não possuem em sua casa pessoas que fumam ou fazem uso de álcool, somente $3(10 \%)$ possuem pessoas que fazem o uso de álcool e 7 (23,3\%) possuem pessoas que utilizam tabaco. Ao serem questionadas sobre o uso de tabaco, 25 gestantes (83,3\%) não fumaram durante a gestação, 1 (3,3\%) fumou apenas no início e $4(13,3 \%)$ continuam fumando. Destas que fumam, as quantidades informadas foram: 5, 6, 10 e 15 unidades/dia. Em relação ao uso de álcool e drogas, 28 gestantes (93,3\%) informaram que não usam e $2(6,7 \%)$ consumiram cerveja durante a gestação.

Sobre o número de gestações, predominam mulheres que tiveram 1 ou 2 gestações (63,3\%). Ainda, $23(76,7 \%)$ informaram que não tiveram aborto, enquanto $7(23,3 \%)$ confirmaram o fato. Destas, 6 mulheres tiveram somente 1 aborto, e 1 mulher abortou 2 vezes. Em relação ao planejamento da gestação, 16 (53,3\%) indicaram que sim e $14(46,7 \%)$ indicaram que não. Todos os resultados seguem na Tabela 2.

Tabela 2 - Comportamento durante a gestação. Unidade Básica de Saúde, Flores da Cunha, RS, Brasil, 2021 $(\mathrm{N}=30)$.

\begin{tabular}{lrr}
\hline & n & $\%$ \\
\hline Na sua casa, alguém faz uso de & & \\
\hline Álcool & 3 & 10,0 \\
Tabaco & 7 & 23,3 \\
Nenhum & 20 & 66,7 \\
\hline Fumou no período gestacional & & \\
\hline Sim & 4 & 13,3 \\
Não & 25 & 83,3 \\
Somente no início & 1 & 3,3 \\
\hline Se sim, quantos cigarros por dia & & \\
\hline 5 cigarros & 1 & 3,3 \\
6 cigarros & 1 & 3,3 \\
10 cigarros & 1 & 3,3 \\
15 cigarros & 1 & 3,3 \\
\hline
\end{tabular}




\begin{tabular}{lrr}
\hline Uso de álcool e droga & & \\
\hline Sim & 2 & 6,7 \\
Não & 28 & 93,3 \\
\hline Qual & 2 & 6,7 \\
\hline Cerveja & & \\
\hline Número de gestações & 5 & 16,7 \\
\hline Nenhuma gestação anterior & 7 & 23,3 \\
1 gestação & 12 & 40,0 \\
2 gestações & 3 & 10,0 \\
3 gestações & 2 & 6,7 \\
4 gestações & 1 & 3,3 \\
6 gestações & & \\
\hline Aborto & 7 & 23,3 \\
\hline Sim & 23 & 76,7 \\
\hline Não & & \\
\hline Se sim, quantos & 6 & 20,0 \\
\hline 1 aborto & 1 & 3,3 \\
\hline abortos & & \\
\hline Gravidez planejada & 16 & 53,3 \\
\hline Sim & 14 & 46,7 \\
\hline Não & & \\
\hline & & \\
\hline
\end{tabular}

Com relação ao tempo de gestação, as semanas são variadas, com destaque para 37 semanas $(23,3 \%), 33$ semanas $(20 \%)$ e 39 semanas $(16,7 \%)$. Sobre o recebimento de orientação quanto ao tipo de parto, 18 gestantes (6o\%) indicaram que receberam, enquanto $12(40 \%)$ não receberam. Quanto ao tipo de parto orientado, $14(46,7 \%)$ informaram o parto normal, 6 (20\%) o parto cesárea por necessidade e $5(16,7 \%)$ o parto cesárea por opção.

Quando perguntado à gestante sobre a sua vontade, 20 mulheres $(66,7 \%)$ indicaram que preferem o parto normal e 10 (33,3\%) a cesárea. As justificativas mencionadas para o parto normal foram: recuperação mais rápida $(20 \%)$, melhor recuperação da mãe e do bebê (10\%), sentimento de tranquilidade (3,3\%) e experiência positiva com o parto anteriormente (3,3\%). Já os pretextos citados para a cesárea foram: experiência positiva com o parto anteriormente (10\%), experiência ruim com o parto normal $(3,3 \%)$, sentimento de tranquilidade $(3,3 \%)$, menos sofrimento $(3,3 \%)$ e planejamento de ligadura (3,3\%), conforme mostra a Tabela 3.

Tabela 3 - Tipos de partos. Unidade Básica de Saúde, Flores da Cunha, RS, Brasil, $2021(\mathrm{~N}=30)$.

\begin{tabular}{|c|c|c|}
\hline & $\mathbf{n}$ & $\%$ \\
\hline \multicolumn{3}{|l|}{ Semanas de gestação } \\
\hline 31 semanas & 2 & 6,7 \\
\hline 32 semanas & 3 & 10,0 \\
\hline 33 semanas & 6 & 20 \\
\hline 34 semanas & 4 & 13,3 \\
\hline 36 semanas & 1 & 3,3 \\
\hline 37 semanas & 7 & 23,3 \\
\hline 38 semanas & 2 & 6,7 \\
\hline 39 semanas & 5 & 16,7 \\
\hline \multicolumn{3}{|l|}{ Orientação ao tipo de parto } \\
\hline Sim & 18 & 60,0 \\
\hline Não & 12 & 40,0 \\
\hline \multicolumn{3}{|l|}{ Tipo de parto orientado } \\
\hline Normal & 14 & 46,7 \\
\hline Cesárea por opção & 5 & 16,7 \\
\hline Cesárea por necessidade & 6 & 20,0 \\
\hline Sem orientação & 5 & 16,7 \\
\hline \multicolumn{3}{|l|}{ Tipo de parto que gostaria } \\
\hline Normal & 20 & 66,7 \\
\hline Cesárea & 10 & 33,3 \\
\hline \multicolumn{3}{|l|}{ Motivo pelo parto normal } \\
\hline Recuperação mais rápida & 6 & 20,0 \\
\hline Sentimento de tranquilidade & 1 & 3,3 \\
\hline Melhor recuperação da mãe e do bebê & 3 & 10,0 \\
\hline Experiência positiva com o parto normal & 1 & 3,3 \\
\hline \multicolumn{3}{|l|}{ Motivo pelo parto cesárea } \\
\hline Sentimento de tranquilidade & 1 & 3,3 \\
\hline Experiência ruim com o parto normal & 1 & 3,3 \\
\hline Experiência positiva com a cesárea & 3 & 10,0 \\
\hline Menos sofrimento & 1 & 3,3 \\
\hline Irá fazer ligadura & 1 & 3,3 \\
\hline
\end{tabular}


Ao serem questionadas sobre a existência de complicações durante o parto, $23(76,7 \%)$ alegaram que não tiveram nenhuma complicação, enquanto $7(23,3 \%)$ confirmaram que sim. Entre as complicações informadas, 3 (10\%) apresentou infecção do trato urinário, $2(6,6 \%)$ sífilis, $1(3,3)$ anemia gestacional e $1(3,3 \%)$ sangramento. Das 7 gestantes que tiveram complicações, todas afirmaram que realizaram corretamente o tratamento para a complicação ou para a doença no período gestacional.

Sobre os fatores que contribuem para adesão ao pré-natal, 19 gestantes (63,3\%) indicaram que acham importante realizá-lo. De forma complementar, todas gestantes informaram que acham importante a realização do prénatal e os motivos foram: acompanhar a saúde e desenvolvimento do bebê (30\%), acompanhar a saúde e desenvolvimento da mãe e do bebê $(16,7 \%)$, sanar todas as dúvidas (3,3\%) e para a resolução de algum problema $(3,3 \%)$.

Em relação à quantidade de consultas, a maioria possui de 6 a 8 consultas $(66,7 \%)$. A seguir na Tabela 4 apresenta-se todos os resultados.

Tabela 4 - Pré-natal. Unidade Básica de Saúde, Flores da Cunha, RS, Brasil, $2021(\mathrm{~N}=30)$.

\begin{tabular}{lrr}
\hline & n & $\%$ \\
\hline Complicação no período gestacional & & \\
\hline Sim & 7 & 23,3 \\
Não & 23 & 76,7 \\
\hline Se sim, quais complicações & & \\
\hline Anemia gestacional & 1 & 3,3 \\
Infecção do trato urinário & 3 & 10,0 \\
Sífilis & 2 & 6,6 \\
Sangramento & 1 & 3,3 \\
\hline Realizou tratamento correto para a & & \\
complicação & \multicolumn{2}{c}{23,3} \\
\hline Sim
\end{tabular}

\begin{tabular}{lrr}
\hline $\begin{array}{l}\text { Fatores que contribuíram para aderir } \\
\text { ao pré-natal }\end{array}$ & & \\
\hline Qualidade do atendimento & 3 & 10 \\
Proximidade da UBS com a residência & 2 & 6,7 \\
Programa totalmente gratuito & 19 & 63,3 \\
\hline É importante realizá-lo
\end{tabular}

Acha importante realizar o pré-natal

\begin{tabular}{lll}
\hline Sim & $30 \quad 100,0$
\end{tabular}

Por quê?

Acompanhar saúde e desenvolvimento do bebê

930,0

Acompanhar saúde e desenvolvimento da mãe e do bebê

$5 \quad 16,7$

Sanar todas as dúvidas

$1 \quad 3,3$

$\begin{array}{lll}\text { Resolução de algum problema } & 1 & 3,3\end{array}$

\begin{tabular}{lll} 
Não responderam & 14 & 46,7 \\
\hline
\end{tabular}

Quantidade de consultas de pré-natal

realizadas

\begin{tabular}{lrr}
\hline 2 consultas & 2 & 6,7 \\
3 consultas & 1 & 3,3 \\
5 consultas & 1 & 3,3 \\
6 consultas & 8 & 26,7 \\
7 consultas & 4 & 13,3 \\
8 consultas & 8 & 26,7 \\
9 consultas & 3 & 10,0 \\
10 consultas & 3 & 10,0 \\
\hline
\end{tabular}

Aúltimapartedapesquisaquestionousobre os cuidados da equipe de enfermagem e equipe multidisciplinar. Com relação ao atendimento dos enfermeiros durante as consultas, se a gestante tirou as dúvidas, foi bem acolhida, ficou à vontade para conversar, $29(96,7 \%)$ afirmaram que sim. Quanto à percepção da qualidade da equipe de enfermagem, 21 gestantes $(70 \%)$ consideram excelente e 9 (30\%) consideram boa.

Sobre as orientações recebidas pela equipe multidisciplinar, 26 gestantes $(86,7 \%)$ consideram que foram válidas, 3 (10\%) responderam que foram mais ou menos válidas e 1 (3,3\%) informou que não foram válidas. Quanto às orientações que as 
gestantes julgam importantes, 9 (30\%) indicaram o aleitamento materno, $8(26,7 \%)$ a assistência ao recém-nascido, 7 (23,3\%) sinais e sintomas anormais durante o período gestacional e $6(20 \%)$ a prevenção e tratamento de complicações durante a gravidez. Por fim, com relação à qualidade da equipe multidisciplinar, 17 gestantes $(56,7 \%)$ consideram excelente qualidade e 13 (43,3\%) consideram de boa qualidade, conforme apresenta a Tabela 5 .

Tabela 5 - Enfermagem e equipe multidisciplinar. Unidade Básica de Saúde, Flores da Cunha, RS, Brasil, 2021 $(\mathrm{N}=30)$.

\begin{tabular}{|c|c|c|}
\hline & $\mathrm{n}$ & $\%$ \\
\hline \multicolumn{3}{|l|}{$\begin{array}{l}\text { Em relação ao atendimento durante as } \\
\text { consultas com a enfermeira, tirou as } \\
\text { dúvidas, foi bem acolhida, ficou à vontade } \\
\text { para conversar }\end{array}$} \\
\hline Sim & 29 & 96,7 \\
\hline Não & 1 & 3,3 \\
\hline \multicolumn{3}{|l|}{ Qualidade da consulta de enfermagem } \\
\hline Excelente & 21 & 70,0 \\
\hline Boa & 9 & 30,0 \\
\hline \multicolumn{3}{|l|}{$\begin{array}{l}\text { Foram válidas as orientações recebidas } \\
\text { pela equipe multidisciplinar }\end{array}$} \\
\hline Sim & 26 & 86,7 \\
\hline Não & 1 & 3,3 \\
\hline Mais ou menos & 3 & 10,0 \\
\hline \multicolumn{3}{|l|}{ Orientações que são importantes } \\
\hline Orientação sobre o aleitamento materno & 9 & 30,0 \\
\hline $\begin{array}{l}\text { Orientação em relação à assistência ao } \\
\text { recém-nascido }\end{array}$ & 8 & 26,7 \\
\hline $\begin{array}{l}\text { Orientação sobre sinais e sintomas } \\
\text { anormais durante o período gestacional }\end{array}$ & 7 & 23,3 \\
\hline $\begin{array}{l}\text { Orientação em relação à prevenção e } \\
\text { tratamento de complicações durante a } \\
\text { gravidez }\end{array}$ & 6 & 20,0 \\
\hline \multicolumn{3}{|l|}{ Qualidade da equipe multidisciplinar } \\
\hline Excelente & 17 & 56,7 \\
\hline Boa & 13 & 43,3 \\
\hline
\end{tabular}

As questões 24 e 25 do questionário foram desconsideradas, pois se tratava de perguntas em relação aos grupos de gestantes que aconteciam uma vez ao mês e devido a pandemia não está ocorrendo. Estes grupos de gestantes se davam em conjunto com a equipe multidisciplinar, no qual diversos temas eram abordados, todos relacionados à saúde da mulher, gestante, feto, recém-nascido e a puérpera.

\section{DISCUSSÃO}

O presente estudo teve como objetivo identificar a percepção das gestantes quanto ao prénatal, correlacionando com as complicações durante a gestação em uma unidade básica de saúde. Embora a população estudada ter sido predominante por gestantes instruídas, com cor de pele autodeclarada branca e de classe socioeconômica média, o que chama atençãoéa maioria das gestantes serem donas de casa. No entanto, isso contribuiu positivamente para estas aderirem ao programa do pré-natal na atenção básica.

É imprescindível o acompanhamento do pré-natal como elemento de proteção para a mãe e o bebê durante a gestação. A relação entre os cuidados adequados no pré-natal mais os resultados perinatais favoráveis, são de extrema importância, tanto para as puérperas como para os recém-nascidos. A combinação de outros fatores da parentalidade também são determinantes como; as condições socioeconômicas, estado civil, escolaridade, idade materna, número de gestações, podem afetar diretamente na promoção de saúde da mulher, por conseguinte, repercutir na comunidade ${ }^{9}$.

$$
\text { Segundo Saldanha }{ }^{10} \text { a condição }
$$
socioeconômica é um dos fatores cruciais que interferem na não procura pelos cuidados 
relacionados à promoção, prevenção e proteção à saúde, por não serem percebidos como importantes na maior parte do tempo. Ao contrário deste contexto as gestantes do estudo, em sua maioria são donas de casa, sendo assim um dos principais motivos positivos para estas aderirem ao programa do pré-natal. Apesar de não terem nenhum vínculo empregatício, a renda familiar não foi afetada, continuando elevada.

O início precoce do acompanhamento do pré-natal é de extrema importância, para que o acesso a diagnósticos e terapias de muitas patologias que comprometem a saúde da mulher e do bebê, como a hipertensão arterial, anemia, diabetes mellitus e infecções por doenças infectocontagiosas como a sífilis e o HIV, sejam descobertas e logo tratadas, não afetando o desenvolvimento do feto" ${ }^{11}$. Durante a gestação poucas gestantes tiveram alguma complicação. As gestantes que apresentavam complicações, relataram que após a descoberta iniciavam o tratamento corretamente.

Em relação ao uso do álcool e do tabaco durante a gestação, as gestantes do estudo estão conscientes das consequências que essas substâncias podem provocar, bem como afetar no desenvolvimento do bebê, durante o período gestacional. No resultado dos dados coletados apresenta-se um número baixo de gestantes que utilizaram tabaco durante a gestação.

Conforme Tamashiro e colegas ${ }^{12}$, o uso de bebidas alcoólicas durante a gestação está associado ao risco elevado de crescimento fetal, anomalias congênitas, aborto, óbito fetal, prematuridade e Síndrome Alcoólica Fetal, como consequência produz alterações tanto no desenvolvimento cognitivo quanto no comportamental. Já o uso do tabagismo aumenta o risco de aborto espontâneo, ruptura prematura das membranas, gravidez ectópica, restrição de crescimento fetal e parto prematuro. Ainda o autor ressalta que pesquisas tanto nacionais como internacionais apontam taxas de diminuição do uso de drogas ilícitas e lícitas durante a gestação, na qual se dá ao fato da mulher estar mais motivada a cessar ou a diminuir o uso dessas drogas, sendo um período propício para os profissionais de saúde realizarem intervenções necessárias para ajudar a gestante, visando a qualidade de vida materno-infantil.

Quanto às orientações sobre o tipo de parto que teriam, a maioria das gestantes relataram que receberam orientação de parto normal. Estas também desejariam o parto normal, pelos seguintes motivos: recuperação mais rápida, melhor recuperação da mãe e do bebê, sentimento de tranquilidade e experiência positiva com o parto vaginal anteriormente. Entringer e colegas $^{13}$ descrevem que há uma demanda elevada das gestantes pelo parto vaginal através do SUS. Existente diversos fatores nos quais estão relacionados a esta escolha, como: a dieta, privacidade, conforto, deambulação, liberdade de escolha da posição no segundo período, uso restrito de episiotomia, apoio dos centros de parto intra-hospitalares, inserção da enfermagem obstétrica nos serviços públicos e a criação de equipes de plantão para a assistência ao parto normal na rede suplementar. No entanto, ressaltase que essa demanda ainda não supera as taxas de cesarianas realizadas pelo SUS.

Cada vez mais as gestantes estão aderindo ao programa de pré-natal na saúde pública, juntamente na estratégia da saúde da família, devido a qualidade da assistência prestada pela equipe de saúde multidisciplinar, em essencial a equipe de enfermagem que está em contato direto com as gestantes. Aponta-se que as gestantes 
estão conscientes da importância de realizar o pré-natal corretamente, para diminuir os índices de mortalidade materno fetal, além de oferecer diversos serviços gratuitamente como consultas, exames e todo o tipo de assistência voltada para a gestante $\mathrm{e}^{14}$.

As gestantes realizaram acima de seis consultas de acompanhamento do pré-natal preconizadas pelo Ministério da Saúde, desta forma estão conscientes da importância de realizá-lo corretamente. Comprova-se a adesão das gestantes ao programa nesta unidade básica de saúde e se destaca como um dos fatores primordiais a qualidade do atendimento prestado pelo serviço de saúde.

Em relação a qualidade do atendimento prestado pela enfermeira, bem como pela equipe multidisciplinar relataram que foram excelentes. Durante as consultas de acompanhamento do pré-natal sanaram todas as dúvidas, foram bem atendidas e acolhidas. As orientações que mais receberam foram sobre o aleitamento materno, assistência ao recém-nascido, sinais e sintomas anormais durante o período gestacional, prevenção e tratamento de complicações durante a gravidez.

Segundo Rocha e $\operatorname{colegas}^{14}$, a equipe de enfermagem é essencial para contribuir para a promoção da saúde e prevenção de doenças durante o pré-natal. Por meio de informações, com destaque na realização de ações educativas nos encontros mensais das gestantes e relato de experiência da maternidade no período gestacional. Então, a mulher será orientada sobre os cuidados com a saúde na gestação, mudanças no organismo, o momento do parto, cuidados com o recém-nascido, a amamentação, com menos riscos de complicações no puerpério e mais sucesso no cuidado com o bebê, principalmente para as primigestas, que estão vivenciando uma nova experiência na sua vida ${ }^{15}$.
Portanto a qualidade da assistência prestada para com essas gestantes tanto da equipe de enfermagem como da equipe multidisciplinar, foi fundamental para que as estas aderissem ao programa do pré-natal. Por fim, neste estudo não foram encontradas gestantes que não aderiram ao pré-natal, mostrando então que o problema desta pesquisa não foi algo característico deste grupo de gestantes participantes.

\section{CONCLUSÃO}

A pesquisa evidenciou que a realização do pré-natal é necessária e de suma importância para prevenir possíveis complicações durante o período gravídico-puerperal. Na qual a qualidade da assistência prestada tanto pela equipe de enfermagem como pela equipe multidisciplinar desde a primeira consulta do pré-natal, foi essencial para que estas gestantes aderissem ao programa.

É nítido que a percepção e a adesão das gestantes ao pré-natal contribuem positivamente, para descobrir complicações precocemente durante o período gravídico-puerperal, visto que iniciaram o pré-natal logo após a descoberta da gravidez. Contudo as poucas gestantes que tiveram complicações neste estudo, após a descoberta da patologia, iniciaram imediatamente o tratamento da mesma, não acarretando danos tanto para mãe, como para o feto. Diante disso, fazem-se necessários mais estudos sobre o tema, com um maior número de pessoas estudadas.

\section{DECLARAÇÃO}

Não existe nenhum conflito financeiro, legal ou político envolvendo terceiros (empresas, governo, instituições privadas e particulares etc.). 


\section{REFERÊNCIAS}

1. Andrade UV, Santos JB, Duarte C. A percepção da gestante sobre a qualidade do atendimento pré-natal em UBS, Campo Grande, MS. Rev. Psic. Saúde. 2019;11(1):53-61. doi: 10.20435/pssa.voio.585.

2. Sehnem GD, de Saldanha LS, Arboit J, Ribeiro AC, de Paula FM. Consulta de pré-natal na atenção primária à saúde: fragilidades e potencialidades da intervenção de enfermeiros brasileiros. Rev. Enferm. Referência. 2020;(1):1-14. doi: 10.12707/RIV19050.

3. Cruz GC, Ruiz PC, Junior OCR, de Sousa AD, Pereira RMO, Barroso C, et al. Métodos de avaliação da qualidade de assistência ao pré-natal no Brasil: revisão integrativa da literatura. Rev. Eletr. Acervo Saúde. 2019;(27):e521-e521. doi: 10.25248/reas.e521.2019.

4. Oliveira IG, Castro LLS, Massena AM, dos Santos LVF, de Sousa LB. Qualidade da consulta de enfermagem na assistência ao pré-natal de risco habitual. Rev. Eletr. Enf. 2017;19(28):1-11. doi:10.5216/ ree.v19.40374.

5. Gomes CBDA, Dias RDS, Silva WGB, Pacheco MAB, Sousa FGMD, Loyola CMD. Consulta de enfermagem no pré-natal: narrativas de gestantes e enfermeiras. Texto \& Contexto Enferm. 2019;28:e20170544. doi: 10.1590/1980-265X-TCE-2017-0544.

6. Tomasi E, Fernandes PAA, Fischer T, Siqueira FCV, Silveira DSD, Thumé E, et al. Qualidade da atenção pré-natal na rede básica de saúde do Brasil: indicadores e desigualdades sociais. Cad. Saúde Públ. 2017;33(3):eoo195815. doi: 10.1590/0102-311Xoo195815.

7. Ministério da Saúde (BR). Qualidade da atenção pré-natal na rede básica de saúde do Brasil: indicadores e desigualdades sociais. Brasília: Editora MS; 2016.

8. Gil, AC. Métodos e técnicas de pesquisa social. 5a ed. São Paulo: Atlas; 1999.

9. Menezes LO, Floriano TVN, Lopes IMD. Impacto do perfil socioeconômico de gestantes e parceiros na avaliação da qualidade do pré-natal. Rev. Eletr. Acervo Saúde. 2021;13(1):e5686-e5686. doi: $10.25248 /$ reas.e5686.2021.

10. Saldanha BL. Dificuldades enfrentadas por gestantes adolescentes em aderir ao pré-natal. Reas. 2020;12(9):e416o. doi: 10.25248/reas.e416o.2020.

11. Mayor MSS, Herrera SDSC, Araujo MQ, dos Santos FM, Arantes RV, de Oliveira NA. Avaliação dos indicadores da assistência pré-natal em unidade de saúde da família, em um município da Amazônia Legal. Rev. Cereus. 2018;10(1):91-100. doi: 10.18605/2175-7275/cereus.v1onıp91-10o. 
12. Tamashiro EM, Milanez HM, Azevedo RCSD. "Por causa do bebê": redução do uso de drogas por gestantes. Rev. Bras. Saúde Mat. Infant. 2020;20(1):313-317. doi: 10.1590/1806-93042020000100017.

13. Entringer AP, Pinto MFT, Gomes MADSM. Análise de custos da atenção hospitalar ao parto vaginal e à cesariana eletiva para gestantes de risco habitual no Sistema Único de Saúde. Ciên. \& Saúde Colet. 2019;24:1527-1536. doi: 10.1590/1413-81232018244.06962017.

14. Rocha AC, Andrade GS. Atenção da equipe de enfermagem durante o pré-natal: percepção das gestantes atendidas na rede básica de Itapuranga-GO em diferentes contextos sociais. Rev. Enferm. Contemp. 2017;6(1):30-41. doi: 10.17267/2317-3378rec.v6i1.1153.

15. Peixoto TB. Adesão da vacinação pela gestante no pré-natal: revisão integrativa. 2017. 
